\title{
INTEGRABILITY, RANDOM MATRICES AND PAINLEVÉ TRANSCENDENTS
}

\author{
N. S. WITTE ${ }^{1}$, P. J. FORRESTER ${ }^{2}$ and CHRISTOPHER M. COSGROVE ${ }^{3}$
}

(Received 27 April, 2000)

\begin{abstract}
The probability that an interval $I$ is free of eigenvalues in a matrix ensemble with unitary symmetry is given by a Fredholm determinant. When the weight function in the matrix ensemble is a classical weight function, and the interval $I$ includes an endpoint of the support, Tracy and Widom have given a formalism which gives coupled differential equations for the required probability and some auxiliary quantities. We summarize and extend earlier work by expressing the probability and some of the auxiliary quantities in terms of Painlevé transcendents.
\end{abstract}

\section{Motivations from physics and mathematics}

Diverse branches of physics and mathematics provide many examples of quantities which are known to have the same statistical properties as the eigenvalues of large random matrices. This is the case for quantum chaos-the study of the quantum mechanics of systems which exhibit chaos at the classical level. Here random matrices have emerged as a paradigm, in that those systems for which the underlying classical mechanics are chaotic are observed to have energy levels with the same statistical properties as the eigenvalues of large random matrices, while systems with integrable classical dynamics do not. Many examples are known from the study of two-dimensional billiard systems-a single particle in a closed domain in two dimensions. One of the most celebrated is the Sinai billiard, which is a square billiard with a hard wall circular scatterer in the centre. Numerical studies show that this system, which is known to be chaotic at the classical level, has energy levels with the same statistical properties as those of large real symmetric matrices (see for example [2]). The

\footnotetext{
'Department of Mathematics and Statistics and School of Physics, University of Melbourne, VIC 3010, Australia; e-mail: nsw@ms.unimelb.edu.au.

${ }^{2}$ Department of Mathematics and Statistics, University of Melbourne, VIC 3010, Australia.

${ }^{3}$ School of Mathematics and Statistics, University of Sydney, Sydney NSW 2006, Australia.

(C) Australian Mathematical Society 2002, Serial-fee code 1446-1811/02
} 
status of our understanding is reflected in a conjecture by Bohigas-Giannoni-Schmit which states:

"The spectra of time-reversal invariant systems whose classical analogues are K-systems show the same fluctuation properties as predicted by the GOE."

Here GOE refers to the Gaussian orthogonal ensemble of random real symmetric matrices and K-systems are the most strongly mixing classical systems. The time reversal symmetry is responsible for the requirement that the random matrices have real entries.

Another fascinating and enigmatic connection is that of random matrix theory and number theory, which concerns the statistics of the large zeros of the Riemann zeta function on the critical line. Incredible numerical evidence of Odlyzko [6] based on the calculation of the critical zeros well into the asymptotic regime (zero number $10^{20}$ and $10^{6}$ of its neighbours) has convincingly shown their statistics to accurately follow the Gaussian unitary ensemble (GUE) of random Hermitian matrix predictions. This connection was first observed in analytic work of Montgomery [5] on the pair correlations of the zeros. Denoting

$$
s_{n}=1 / 2+i E_{n}, \quad d(E)=\sum_{n \geq 1} \delta\left(E-E_{n}\right),
$$

for the zeros and their density respectively, the asymptotic pair correlation was evaluated as

$$
1+S(\tilde{\epsilon}) \equiv \lim _{E \rightarrow \infty} \frac{\langle d(E) d(E+\epsilon)\rangle}{\langle d(E)\rangle\langle d(E+\epsilon)\rangle}=\delta(\tilde{\epsilon})+1-\frac{\sin ^{2} \pi \tilde{\epsilon}}{(\pi \tilde{\epsilon})^{2}}=1+S_{\mathrm{GUE}}(\tilde{\epsilon})
$$

where $\tilde{\epsilon} \equiv \epsilon /\langle d(E)\rangle$. This prediction, and other statistical measures of the asymptotic zeros such as spacing statistics, were tested in the numerical work of Odlyzko with almost perfect agreement being found at a graphical level.

\section{Matrix ensembles and statistical mechanics of levels}

We take as an example of a random matrix ensemble the GUE, which in application to chaotic quantum systems applies in the case of no time-reversal symmetry $[H, T] \neq 0$. One constructs a $N \times N$ Hermitian matrix $X$ by taking real diagonal elements $x_{j j}$ to be independently chosen with probability distribution function (p.d.f.)

$$
\frac{1}{\sqrt{\pi}} e^{-x_{i j}^{2}}
$$


and upper triangular elements $x_{j k} \equiv u_{j k}+i v_{j k}$ are independently chosen with p.d.f.

$$
\frac{2}{\pi} e^{-2\left(u_{j k}^{2}+v_{j k}^{2}\right)}=\frac{2}{\pi} e^{-2\left|x_{j k}\right|^{2}} .
$$

The joint p.d.f. for elements of $X$ is then

$$
P(X) \propto \prod_{j=1}^{N} e^{-x_{j j}^{2}} \prod_{1 \leq j<k \leq N} e^{-2\left|x_{j k}\right|^{2}}=\prod_{1 \leq j, k \leq N} e^{-\left|x_{j}\right|^{2}}=e^{-\operatorname{Tr}\left(X^{2}\right)}
$$

This distribution function is invariant under arbitrary unitary transformations

$$
P\left(U^{-1} X U\right)=P(X)
$$

and hence the naming of the ensemble. The Gaussian form of the p.d.f. can be uniquely characterised by the invariance (6) and the factorisation property

$$
P(X)=\prod_{j, k} f\left(x_{j k}\right) .
$$

Alternatively it is the unique p.d.f. which maximises the entropy

$$
S[P]=-\int \mu(d X) P \log P,
$$

subject to the constraint $\left\langle\operatorname{Tr} X^{2}\right\rangle=N^{2}$. Primary interest centres on the eigenvalue level statistics and so a description in terms of eigenvalues is required. This is achieved by the mapping

$$
N(N+1) / 2 \text { elements }\left\{x_{i j}\right\} \rightarrow \begin{cases}N \text { eigenvalues } & \lambda_{1} \leq \cdots \leq \lambda_{N} \\ N(N-1) / 2 \text { variables } & p_{1}, \ldots\end{cases}
$$

where the $p_{i}$ are related to the eigenvectors. Using the transformation of Hermitian matrices to the diagonal representation, the volume form is correspondingly transformed as

$$
(d X) \equiv \bigwedge_{j=1}^{N} d x_{j j} \bigwedge_{1 \leq j<k \leq N} d u_{j k} \wedge d v_{j k}=\left(U^{\dagger} d U\right) \prod_{1 \leq j<k \leq N}\left(\lambda_{j}-\lambda_{k}\right)^{2} \bigwedge_{j=1}^{N} d \lambda_{j}
$$

This eigenvalue and eigenvector dependence thus factorises. The eigenvalue p.d.f. can then be written as

$$
P\left(\lambda_{1}, \ldots, \lambda_{N}\right)=\frac{1}{C_{N}} e^{-\sum_{j} \lambda_{j}^{2}} \prod_{1 \leq j<k \leq N}\left(\lambda_{j}-\lambda_{k}\right)^{2} .
$$


This p.d.f. is proportional to the Boltzmann weight factor

$$
p\left(x_{1}, \ldots, x_{N}\right)=\frac{1}{C_{\beta N}} \prod_{j=1}^{N} w\left(x_{j}\right) \prod_{1 \leq j<k \leq N}\left|x_{j}-x_{k}\right|^{\beta}=\frac{1}{Z_{N}} e^{-\beta U\left(x_{1}, \ldots, x_{N}\right)},
$$

from equilibrium statistical mechanics for a system of $N$ charges on the line (or circle) at temperature $1 / \beta$ located at positions $x_{j}$, and with the total potential energy

$$
U\left(x_{1}, \ldots, x_{N}\right)=-\frac{1}{\beta} \sum_{1 \leq j \leq N} \ln w\left(x_{j}\right)-\sum_{1 \leq j<k \leq N} \ln \left|x_{j}-x_{k}\right|
$$

(with $-\log w(x)=x^{2}$ ) composed of a 1-body confining potential (the first term) and a 2-body electrostatic interaction (the second term). Consequently a configuration integral can be defined as

$$
Z_{N}=\int_{-\infty}^{\infty} d x_{1} \cdots \int_{-\infty}^{\infty} d x_{N} e^{-\beta U}
$$

The gap probability, or probability for the exclusion of eigenvalues from an interval $I$, is a fundamental statistic underlying the spacing distribution and is defined by

$$
E_{\beta}(0 ; I)=\frac{1}{Z_{N}} \int_{i} d x_{1} \cdots \int_{\bar{I}} d x_{N} e^{-\beta U}
$$

with $\bar{I}=(-\infty, \infty) \backslash I$, however this form is often not suitable to work with. Instead we seek a form involving the $n$-particle distribution function

$$
\begin{aligned}
& \rho_{n}\left(x_{1}, \ldots, x_{n}\right) \equiv\left\langle\sum_{1 \leq j_{1} \neq \cdots \neq j_{n} \leq N} \delta\left(x_{1}-x_{j_{1}}\right) \cdots \delta\left(x_{n}-x_{j_{n}}\right)\right\rangle \\
& =\frac{N !}{(N-n) !} \frac{1}{Z_{N}} \int_{-\infty}^{\infty} d x_{n+1} \cdots \int_{-\infty}^{\infty} d x_{N} e^{-\beta U\left(x_{1}, \ldots, x_{N}\right)} .
\end{aligned}
$$

This can be done by introducing the generating functional

$$
Z_{N}[a] \equiv \frac{1}{Z_{N}} \int_{-\infty}^{\infty} d x_{1} \cdots \int_{-\infty}^{\infty} d x_{N} \prod_{l=1}^{N}\left[1+a\left(x_{l}\right)\right] e^{-\beta U\left(x_{1}, \ldots, x_{N}\right)}
$$

expanding out the product and using the definition (16) to obtain the formula

$$
Z_{N}[a]=1+\sum_{n=1}^{\infty} \frac{1}{n !} \int_{-\infty}^{\infty} d x_{1} \ldots \int_{-\infty}^{\infty} d x_{n} \prod_{l=1}^{n} a\left(x_{l}\right) \rho_{n}\left(x_{1}, \ldots, x_{n}\right)
$$


Choosing $-a(x)$ to be the characteristic function of $I$ so that

$$
a(x)= \begin{cases}-1 & \text { if } x \in I \\ 0 & \text { if } x \notin I,\end{cases}
$$

we see that (17) coincides with the definition (15) of $E_{\beta}(0 ; I)$ and (18) then gives

$$
E_{\beta}(0 ; l)=1+\sum_{n=1}^{\infty} \frac{(-1)^{n}}{n !} \int_{I} d x_{1} \ldots \int_{I} d x_{n} \rho_{n}\left(x_{1}, \ldots, x_{n}\right)
$$

The probability $E_{\beta}(0 ; I)$ determines the p.d.f. that given there is a particle at $a_{1}$, the nearest neighbour to the right is at $a_{2}$, through the formula

$$
\begin{aligned}
p_{\beta}\left(0 ;\left(a_{1}, a_{2}\right)\right) & \equiv \frac{N(N-1)}{\rho\left(a_{1}\right) Z_{N}} \int_{\bar{I}} d x_{3} \cdots \int_{\bar{I}} d x_{N} e^{-\beta U\left(a_{1}, a_{2}, x_{3}, \ldots, x_{N}\right)} \\
& =-\frac{1}{\rho\left(a_{1}\right)} \frac{\partial^{2}}{\partial a_{1} \partial a_{2}} E_{\beta}\left(0 ;\left(a_{1}, a_{2}\right)\right)
\end{aligned}
$$

The distribution $p_{\beta}\left(0 ;\left(a_{1}, a_{2}\right)\right)$ is readily measured empirically, especially in the bulk region of the spectrum when translation invariance gives $p_{\beta}\left(0 ;\left(a_{1}, a_{2}\right)\right)=p_{\beta}(0$; $\left.\left|a_{1}-a_{2}\right|\right)$.

\section{Fredholm integral operators}

For $\beta=2$ the probability $E_{\beta}(0 ; I)$ can be written as the determinant of a Fredholm integral operator [4]. To show this we make use of the fact that the general $n$-particle distribution has the determinant representation

$$
\rho_{n}\left(x_{1}, \ldots, x_{n}\right)=\operatorname{det}\left[K\left(x_{i}, x_{j}\right)\right]_{i, j=1}^{n},
$$

where, with $\left\{p_{n}(x)\right\}_{n=0,1, \ldots}$ the orthonormal polynomials with respect to the weight function $w(x)$,

$$
K(x, y) \equiv[w(x) w(y)]^{1 / 2} \sum_{n=0}^{N-1} p_{n}(x) p_{n}(y) .
$$

With $a_{n}$ denoting the coefficient of $x^{n}$ in $p_{n}(x)$, the Christoffel-Darboux summation gives

$$
\begin{aligned}
K(x, y) & =\frac{a_{N-1}}{a_{N}}[w(x) w(y)]^{1 / 2} \frac{p_{N}(x) p_{N-1}(y)-p_{N-1}(x) p_{N}(y)}{x-y} \\
& =\frac{\phi(x) \psi(y)-\phi(y) \psi(x)}{x-y} .
\end{aligned}
$$


The determinant formula (22) substituted in (20) gives a well known [8] expansion formula for the determinant of an integral operator in terms of its kernel. Explicitly

$$
E(0 ; I)=\operatorname{det}(\mathbb{Q}-\mathbb{K})
$$

where $\mathbb{K}$ is the integral operator on $I$ with kernel $K(x, y)$.

Tracy and Widom [7] have shown that by introducing a number of auxiliary quantities related to $\mathbb{K}$, for some special choices of $w(x)$ and $I$ the Fredholm determinant (25) can be specified in terms of the solution of a nonlinear equation. The auxiliary quantities required are the operators

$$
\frac{\mathbb{K}}{\mathbb{Z}-\mathbb{K}} \doteq R(x, y), \quad \frac{\mathbb{Q}}{\mathbb{Q}-\mathbb{K}} \doteq \rho(x, y),
$$

where the notation $\mathbb{A} \doteq A(x, y)$ denotes that the integral operator $\mathbb{A}$ has kernel $A(x, y)$; the pair of functions

$$
\begin{aligned}
Q(x) & =\int_{I} d y \rho(x, y) \phi(y) \doteq(\mathbb{l}-\mathbb{K})^{-1} \phi, \\
P(x) & =\int_{I} d y \rho(x, y) \psi(y) \doteq(\mathbb{Q}-\mathbb{K})^{-1} \psi, \\
q_{j} & \equiv \lim _{x \rightarrow a_{j}} Q(x), \quad p_{j} \equiv \lim _{x \rightarrow a_{j}} P(x) ;
\end{aligned}
$$

and the scalar products

$$
\begin{aligned}
& u=\langle\phi \mid Q\rangle=\int_{I} d y Q(y) \phi(y), \\
& v=\langle\psi \mid Q\rangle=\int_{I} d y Q(y) \psi(y)=\langle\phi \mid P\rangle=\int_{I} d y P(y) \phi(y), \\
& w=\langle\psi \mid P\rangle=\int_{I} d y P(y) \psi(y) .
\end{aligned}
$$

The essence of the Tracy and Widom machinery in this setting is to set up a system of partial differential equations for the above quantities with respect to the endpoints of the interval, defined by $I=\left(a_{1}, a_{2}\right)$. The equations in one set are universal while a second set of differential equations describes the coincident functions and their derivatives, and includes specific features of the particular orthogonal polynomial system at hand. In particular one requires the differential-recurrence relations

$$
\begin{aligned}
m(x) \phi^{\prime}(x) & =A(x) \phi(x)+B(x) \psi(x), \\
m(x) \psi^{\prime}(x) & =-C(x) \phi(x)-A(x) \psi(x),
\end{aligned}
$$


TABLE 1. Painlevé transcendents in the Gaussian Unitary Ensemble.

\begin{tabular}{|c|}
\hline $\begin{array}{c}\text { Gaussian weights and Hermite orthogonal polynomials } \\
w(s)=e^{-s^{2}}, m(s)=1, A(s)=-1, B(s)=C(s)=\sqrt{2 N}\end{array}$ \\
\hline$I=(s, \infty)$ \\
\hline$\sqrt{2 N}[u-w]+2 u w=q p$ \\
\hline$E_{2}(s)=\exp \left(-\int_{s}^{\infty} d t R(t)\right)$ \\
\hline$R(s) \stackrel{s \rightarrow \infty}{\sim}\left(2^{N-1} / \sqrt{\pi}(N-1) !\right) s^{2 N-2} e^{-s^{2}}$ \\
$q^{\prime} / q^{s \rightarrow \infty}-s+N / s \quad p^{\prime} / p \stackrel{s \rightarrow \infty}{\sim}-s+(N-1) / s$ \\
\hline$\omega(s)$ PIV $\alpha=2 N-1, \beta=0$ \\
\hline$q^{\prime} / q=-s-\omega-2 N \omega /\left(\omega^{\prime} / 2-\omega^{2} / 2-s \omega\right)$ \\
$p^{\prime} / p=-\omega / 2+\omega^{\prime} /(2 \omega)$ \\
$R=-\left(s^{2}-2 N\right) \omega / 2-s \omega^{2} / 2-\omega^{3} / 8+\omega^{2} /(8 \omega)$ \\
\hline$\omega(s)$ PIV $\alpha=-N \pm 1, \beta=-2 N^{2}$ \\
\hline$R=-N^{2} /(2 \omega)-N s-\left(s^{2}+N\right) \omega / 2-s \omega^{2} / 2-\omega^{3} / 8+\omega^{2} /(8 \omega)$ \\
\hline
\end{tabular}

where $m, A, B, C$ are polynomials in $x$ parameterised by

$$
\begin{array}{ll}
m(x)=\mu_{0}+\mu_{1} x+\mu_{2} x^{2}, & A(x)=\alpha_{0}+\alpha_{1} x, \\
B(x)=\beta_{0}+\beta_{1} x, & C(x)=\gamma_{0}+\gamma_{1} x,
\end{array}
$$

which is a common structure for the classical orthogonal polynomials.

\section{Spacing p.d.f. and Painlevé transcendents}

The classical weights are

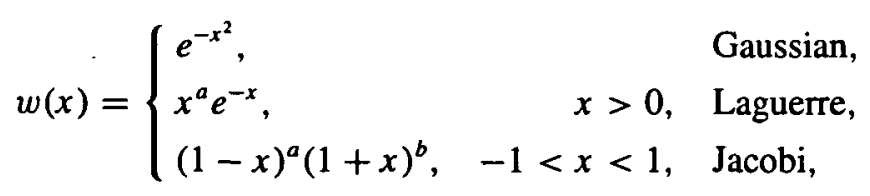

which together with their corresponding orthonormal polynomials give rise to functions $\phi(x), \psi(x)$ satisfying (30) and (31). For general parameters in all these cases, it has only been possible to characterise $E_{2}(0 ; I)$ in terms of a nonlinear equation when the interval $I$ includes an endpoint of the support of $w(x)$ and so depends only on one variable. It turns out that then the auxiliary quantities $q^{\prime} / q, p^{\prime} / p$ and $R \equiv R(s, s)$, as well as the probability $E_{2}(0 ; l)$, can be expressed in terms of Painlevé transcendents.

In Tables 1-3 we list the evaluations for the three classical weights respectively. Also listed are the specific coefficients (31), and an equation or equations (integrals of motion) relating the quantities. 
TABLE 2. Painlevé transcendents in the Laguerre Unitary Ensemble.

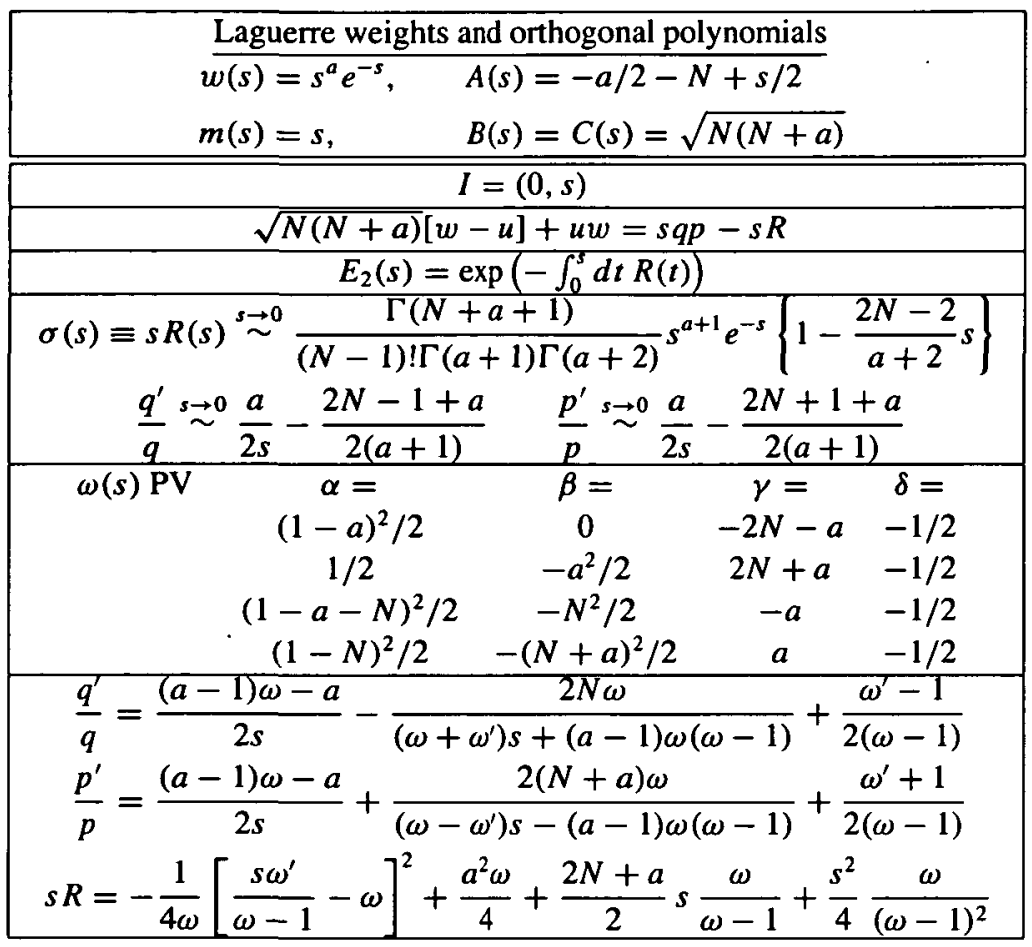

To illustrate the construction of these tables, consider the Jacobi weight with $I=(-1, s)$. Direct application of the Tracy and Widom theory gives the coupled differential equations

$$
\begin{gathered}
{\left[\ln E_{2}\right]^{\prime}=-R,} \\
\left(1-s^{2}\right) q^{\prime}=\left[\alpha_{0}+\alpha_{1} s+v\right] q+\left[\beta_{0}+u\left(2 \alpha_{1}-1\right)\right] p, \\
\left(1-s^{2}\right) p^{\prime}=-\left[\gamma_{0}-w\left(2 \alpha_{1}+1\right)\right] q-\left[\alpha_{0}+\alpha_{1} s+v\right] p, \\
u^{\prime}=q^{2}, \quad v^{\prime}=q p, \quad w^{\prime}=p^{2}, \\
\left(1-s^{2}\right) R=\left[\gamma_{0}-w\left(2 \alpha_{1}+1\right)\right] q^{2}+\left[\beta_{0}+u\left(2 \alpha_{1}-1\right)\right] p^{2}+\left[\alpha_{0}+\alpha_{1} s+v\right] 2 q p, \\
{\left[\left(1-s^{2}\right) R\right]^{\prime}=2 \alpha_{1} q p .}
\end{gathered}
$$

From this system of differential equations two integrals can be constructed [9], which are given in Table 3, and using these a sequence of eliminations leads to a second degree second order differential equation for $\sigma(s) \equiv\left(1-s^{2}\right) R(s)$. This was first found in an indirect manner in [3]. In Table 3 we identify the solution of this equation with a number of P-VI transcendents, and give that specific mapping in the first case listed. For this purpose we use theory from [1]. 
TABLE 3. Painleve transcendents in the Jacobi Unitary Ensemble.

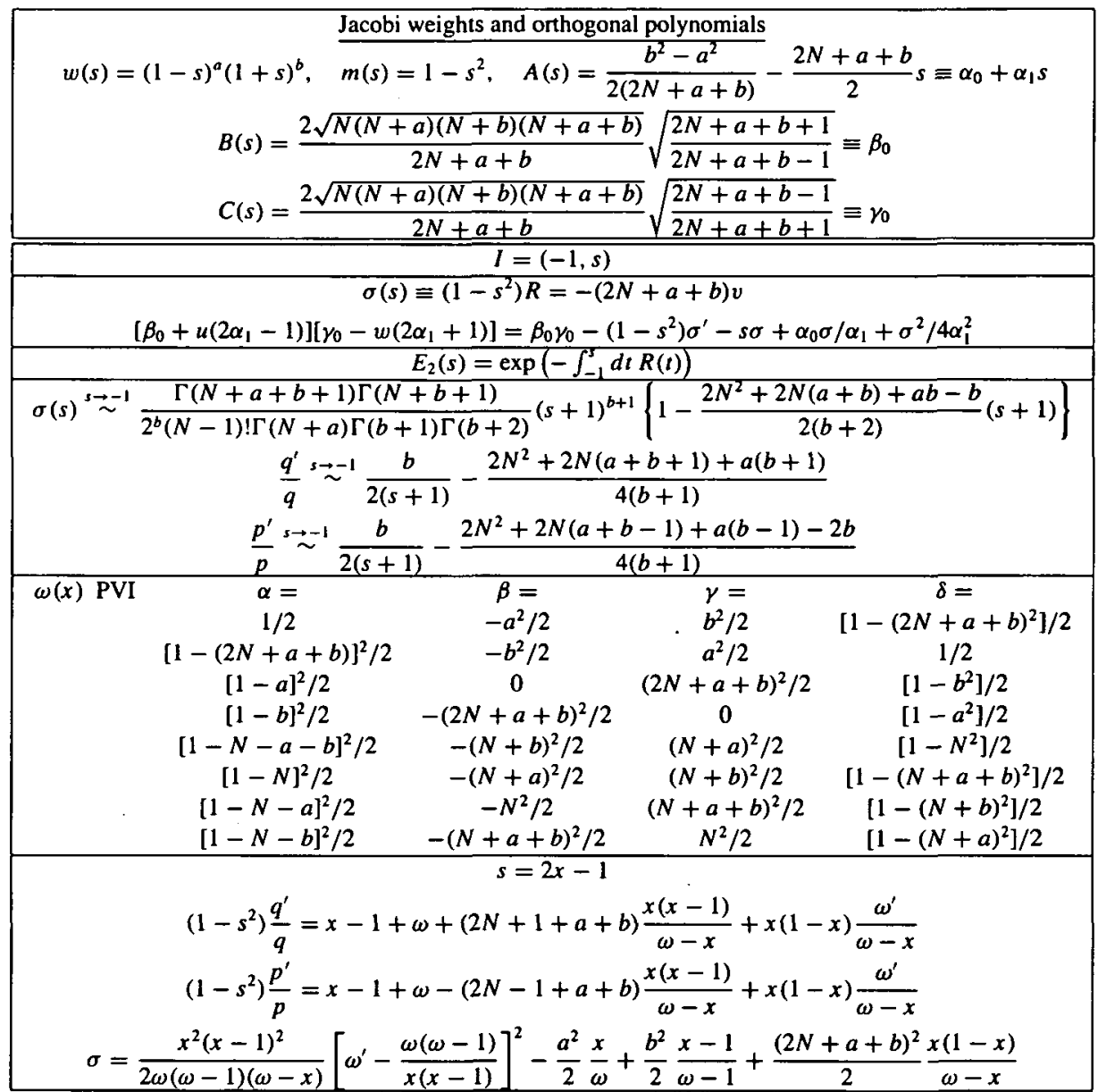

\section{Conclusions}

In this work we have considered the evaluation of the gap probability $E_{2}(0 ; I)$ for the classical weights (32) with $l$ including an endpoint of the support. This quantity, and the auxiliary quantities $p^{\prime} / p, q^{\prime} / q$ and $R \equiv R(s, s)$, are given in terms of Painlevé transcendents thereby extending findings of earlier works $[3,7,9]$. Our approach has been to construct all the integrals of the motion which allows us to reduce the system of differential equations arising in the Tracy and Widom formalism to a single second order ODE by the most direct path possible. Furthermore we have displayed the appearance of the Painlevé transcendents in the random matrix context in a transparent manner, which we consider to be essential for an understanding of the 
relevance of integrability in this situation.

\section{Acknowledgement}

This paper is dedicated to Martin Kruskal on the occasion of his 75th birthday.

\section{References}

[1] C. M. Cosgrove and G. Scoufis, "Painlevé classification of a class of differential equations of the second order and second degree", Stud. Appl. Math. 88 (1993) 25-87.

[2] T. Guhr, A. Müller-Groeling and H. A. Weidenmüller, "Random-matrix theories in quantum physics: common concepts", Phys. Rep. 299 (1999) 189-425.

[3] L. Haine and J.-P. Semengue, "The Jacobi polynomial ensemble and the Painlevé VI equation", $J$. Math. Phys. 40 (1999) 2117-2134.

[4] M. L. Mehta, Random matrices, 2nd ed. (Academic Press, San Diego, 1991).

[5] H. L. Montgomery, "The pair correlation of zeros of the zeta function", in Proc. Sympos. Pure Math. vol. 24, (Amer. Math. Soc., Providence, RI, 1973) 181-193.

[6] A. M. Odlyzko, "The $10^{20}$ th zero of the Riemann zeta function and 70 million of its neighbours", preprint, AT\&T Bell Laboratories, Murray Hill, NJ 07974, 1989.

[7] C. A. Tracy and H. Widom, "Fredholm determinants, differential equations and matrix models", Commun. Math. Phys. 163 (1994) 33-72.

[8] E. T. Whittaker and G. N. Watson, A course of modern analysis, 2nd ed. (Cambridge University Press, Cambridge, 1965).

[9] N. S. Witte and P. J. Forrester, "Gap probabilities in the finite and scaled Cauchy random matrix ensembles", Nonl. 13 (2000) 1965-1986. 